\title{
Wee beasties
}

\section{How a tiny observation turned out to have a controlling influence.}

\section{Paul Nurse}

A major turning point in my scientific life involved not only a group of individuals (important as they were for what happened), but also a microorganism - or to be more precise, a mutant microorganism. The time was 1974 and the place was Murdoch Mitchison's laboratory in the zoology department at the University of Edinburgh. Mitchison had been interested in the celldivision cycle since the 1950s, working mainly with the fission yeast Schizosaccharomyces pombe. $\mathrm{He}$ recognized that the length of this rod-shaped, single-celled organism was a good marker for how far the cell cycle had progressed, which made fission yeast a convenient model for cell cycle studies. It was this microorganism which transformed my scientific life.

The previous year, I had been a graduate student in Norwich studying amino-acid pools in fungi. This project required me to nurture a rather temperamental Beckman amino-acid analyser, and I often found myself keeping this machine company throughout the night — just to make sure it kept running! During these long night vigils, I read numerous research papers to keep me awake and two from Lee Hartwell attracted my attention. These described the isolation of temperature-sensitive cell-division-cycle (cdc) mutants in budding yeast (Saccharomyces cerevisiae), which failed to progress through the cell cycle when grown at a high temperature. Immediately, I decided that I wanted to do something similar and, being familiar with Mitchison's work, thought fission yeast would be a good organism for this project. I contacted Mitchison, who was enthusiastic about me coming to Edinburgh, but wisely suggested that I should first learn and become familiar with genetic techniques. So I went to Urs Leupold in Bern, where he and his colleague, Peter Munz, taught me fission-yeast genetics. I finally arrived in Edinburgh at the start of 1974, with my newly acquired (but rather limited) genetic knowledge, and started isolating cdc mutants in fission yeast.

However, the isolation procedure was very laborious and so progress was very slow. First, temperature-sensitive mutants (those unable to form colonies at a high temperature) had to be identified by replica plating yeast colonies from a low to a high temperature.

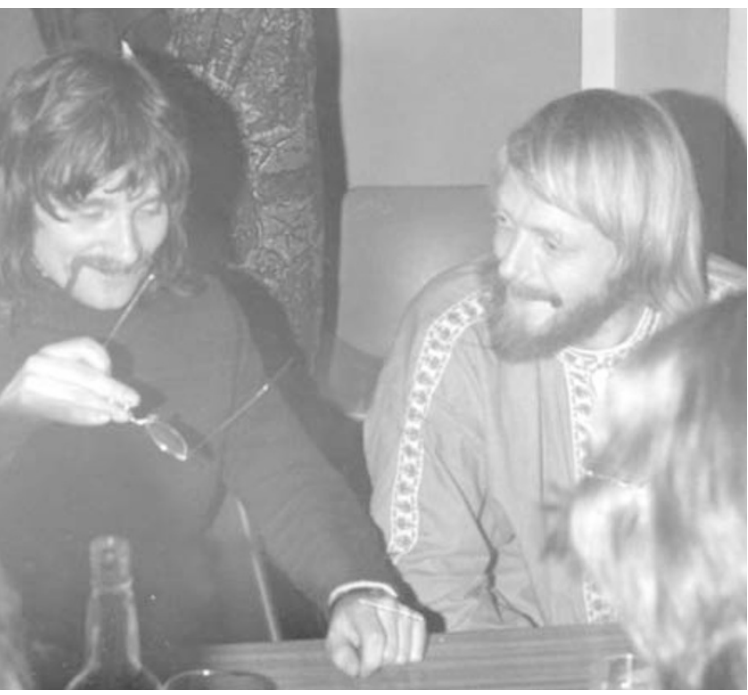

before they had grown to their normal size.

I called over my friend and colleague, Pierre Thuriaux, who was visiting from Bern. He was aware of my limited genetic skills and gently suggested that my plates were contaminated with another microorganism. It was another day before we and Mitchison were convinced that it was really fission yeast, albeit a small one. During our conversations we recalled a seminar from a colleague in the department of genetics, Henry Kacser, on the control of metabolic flux. Kacser had argued that although control was distributed through all steps of a metabolic pathway, some steps could be more rate-
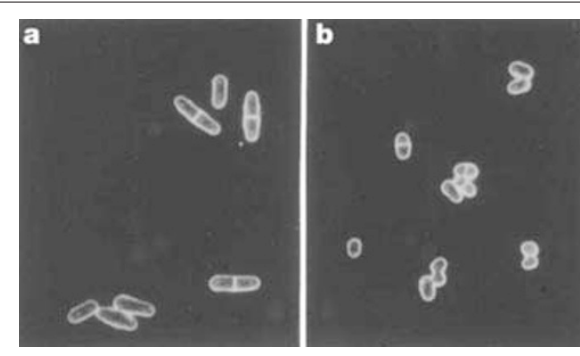

Next, these mutants were screened visually using a microscope to find colonies that contained elongated cells, which indicated that they could not complete their cell cycle and divide, although they continued to grow. By the end of the year I had only found approximately 30 cdc mutants.

I devised an enrichment procedure to try and speed things up. I centrifuged cell populations through a density gradient to separate large, elongated cells from normalsized cells. The larger cells, some of which would be cdc mutants, should make it to the bottom of the gradient, and these fractions were plated out. After isolating a few mutants this way, something strange happened. There was a clump of cells in the middle of the microscope field that seemed to be smaller than normal. My first response was disbelief - how could such small cells have got to the bottom of the gradient? This thought was slowly replaced by another one - what was the significance of these tiny cells? Fission yeast cells usually divide at a constant cell length, so if these cells were shorter than normal, this must mean that they had divided at a small size. This was only possible if cells had progressed more rapidly through their cycle, dividing prematurely limiting than others. Was it possible that this small-sized mutant (soon to be called 'wee' to reflect its Scottish origin) had a defective gene for a major rate-limiting step in the pathways controlling cell-cycle progression? This simple interpretation turned out to be correct when it was eventually demonstrated that Wee1 regulates the cyclin-dependent kinase that acts as the major engine of the fission-yeast cell cycle.

What lessons did I learn from this turning point in my career? The first was the importance in biology of letting the organism you are studying guide you in the right direction to solve your problem. By using microscopic visual inspection, I was not being too restricted in my search for mutants. If something unexpected turned up, I could see it. This allowed mutants that no one had imagined existing before to be observed and thought about, opening up new avenues of enquiry. Serendipidity was given a chance to play a role, allowing nature to constrain and guide the infinite possibilities that occur to the human mind.

A second lesson was the importance of spending time trying to understand the real biological significance of observations made on cells and organisms before undertaking detailed investigations of the molecular mechanisms involved. Understanding the biology properly is as important as the subsequent mechanistic insight.

One final thought - why were the wee mutant cells at the bottom of the gradient? I don't know, perhaps it was because they clump easily or maybe I was just very lucky. Paul Nurse is in the Rockefeller University, 1230 York Avenue, New York 10021, USA. 